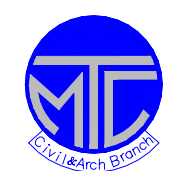

ICCAE

Military Technical College

Kobry Elkobbah, Cairo, Egypt 6h International Conference

On Civil \& Architecture

Engineering

\title{
Implementation of Buried Structure to Withstand Ground Shock from Subsurface Explosion
}

\author{
Louay, S. Mohamed ${ }^{1}$, Hassan, M. Farag ${ }^{2}$, Mohamed, N. Fayed ${ }^{3}$ \\ Abou Zied, M. Tarkhan ${ }^{4}$
}

\begin{abstract}
The present study aims at establishing a fully coupled 3D numerical approach for analyzing the response of buried structure subjected to subsurface blast loading. In this approach, the explosion source, the propagation of stress wave in the soil and the interaction between soil and the structure are integrated into a single model. The validity of proposal model was established by comparison of the obtained numerical results with the available field test. The model is then used to study the effect of medium on buried structure response. Increasing the structural resistance efficiency to withstand ground shock from subsurface explosion was achieved by introducing ground shock attenuation sand layer in the front of wall facing the explosion source. The finite element hydrocode 'AUTODYN' was employed.
\end{abstract}

Keywords: Buried Structure, Subsurface Explosion, Ground Shock, Numerical Simulation

1- Ph.D, Candidate, Syria

2- Ph.D, Egyptian Armed Forces.

3- Professor, Ain Shams University, Egypt

4- Ass. Professor, Helwan University, Egypt 


\section{Introduction}

The processes governing the response of the buried structure subjected to subsurface explosion are very complex, involving dynamic interactions among the explosive, the soil and the underground structure. Major phenomena include the formation of the crater or camouflet by the explosion, the propagation of the shock wave in the medium, and the interaction between medium and the structure. The nonlinear properties and large deformation of the soil and damage in reinforced concrete make the whole process highly nonlinear with respect to the material and geometry [1].

Two numerical methods are usually used to analyze the response of an underground structure under blast loading, namely the uncoupled method and the coupled method [2]. In the uncoupled method, the main physical process is divided into several constitutive phases; the output of one phase is the input of the next phase. In this respect, the problem under consideration can be divided into three phases: (1) the detonation of the charge and the formation of crater; (2) the propagation of blast wave; and (3) the response of the structure. The coupled method can be divided into two categories, namely partial coupled method and full coupled method. In the partial coupled method, the above three phases are reduced to two phases, with either the first two or the last two phases being merged. The full coupled method category combines all three phases together in a single model.

The use of open trenches for vibration isolation is an effective passive approach for reducing the energy transfer from the propagating stress wave to a buried structure. Profound reductions in the transfer of energy have been noted as a result of voids in the wave's path. The general effect of the trench is to cut off the energy transfer, and to introduce significant reflections of waves having opposite signs at the soil-to-trench interface. In other words, a compressive wave reaching that interface will be reflected as a tensile wave, thus interacting with the incoming wave for reducing its effect. Since a trench could be collapsed under the applied shock loads (depending on the stress wave intensity), it is justified to investigate the effect of placing a soft material in the trench as a barrier [3].

\section{Proposed full coupled numerical approach}

In the numerical simulations, the partial differential equations governing the basic physical principles of conservation of mass, momentum, and energy are employed. The equations to be solved are both time and space-dependent and nonlinear in nature. These equations, together with equations of state (EOS) and constitutive models describing material behavior and a set of initial and boundary conditions, define the complete system for blast simulations [4].

The solution over the time domain can be achieved by an explicit method, in which, the solution at a given point in time is explicitly expressed as a function of the system variables and parameters, with no requirement for stiffness and mass matrices. Thus the computing cost at each time step is low but may also require numerous time steps for a complete solution.

There are two major ways of describing the solution over the space domain, based on the relative movement between the material particles and the mesh: one is the Eulerian description, while the other is Lagrangian description [5]. In the Eulerian description, the mesh is fixed in space and different material particles move through it. In the Lagrangian description, the mesh and the material particles coincide. The Eulerian description is suited 
for situations where the mesh may be highly distorted; but modeling of the material boundary conditions such as slippage and contact surface is very difficult. The Lagrangian description is more suitable for situations where the deformation is not large but the effects of interface and free boundaries are significant. This concept leads to a requirement for a numerical technique that allows both Eulerian and Lagrangian solutions in a single simulation with coupling between the different techniques in space and time. Such an approach, wherein different methods may be applied within a single numerical analysis, can provide the "best" solution in terms of accuracy and efficiency.

In the problem under investigation, the two descriptions are used in a single analysis. The Eulerian description is used for detonation of explosive material, while the Lagrangian description is suitable for representation of soil and concrete materials location. To overcome the highly Lagrangian mesh distortion in the vicinity of the charge, the erosion criteria is used with suitable values [4].

The conservation equations, the material models as well as the boundary conditions all describe the whole physical problem. The equations are solved to update the solution in successive time steps. The operational procedure will not be presented in detail but a general computational cycle is illustrated. Figure (1) shows the series calculations that are carried out in each computational time step. Starting at the bottom of the figure the boundary forces are updated and combined with the element forces computed during the previous time cycle. Then the accelerations, velocities and positions are computed from the momentum conservation equations and a further integration. From these values the new element volumes, strain and strain rate are calculated. With the use of material models together with the energy equation the element pressure, stresses and energies are calculated, providing forces for use at the start of the next computational cycle.

\subsection{Material models}

The material models chosen in simulation depend on the physical materials. There are four kinds of materials involved in the problem under investigation, namely the media mass (soil), the concrete mass, the reinforcing steel in the structure, and the high energy explosive material.

\section{(a) Concrete modeling}

The response of concrete under shock loading is a complex nonlinear and ratedependent process. When concrete is subjected to hydrostatic pressure, the relationship between hydrostatic pressure and density becomes non-linear at a certain pressure level. Initially, for low-pressure levels the relationship for pressure and density is linear (elastic loading). With further loading, micro cracking occurs in material. Since concrete is porous, the pores collapse and the material will be compacted; this is termed as the plastic compaction phase. At very high pressure levels, when the concrete is fully compacted (all pores are collapsed), the relationship between pressure and density becomes linear again. The EOS used in this analysis is a combined P-Alpha and a polynomial EOS [2]. The P-Alpha EOS defines the starting point for plastic compaction, and the polynomial EOS defines the compaction phase. In Figure (2) the initial density, $\rho$, is the undisturbed concrete density, and the solid density, $\rho_{s}$, is defined as the density at zero pressure for fully compacted solid. The material behaves elastically until the initial compaction pressure, $p_{\text {crush }}$, is reached; thereafter the plastic compaction phase takes place. 
The RHT [6] constitutive model, implemented into AUTODYN was utilized for concrete. This is a new model for general brittle materials, developed by Riedel, Hiermaier and Thoma. This model contains many features common to various similar constitutive models such as pressure hardening, strain hardening, strain rate hardening, third invariant dependence for compressive and tensile meridians, and cumulative damage (strain softening). The material model uses three strength surfaces: an elastic limit surface, a failure surface and the remaining strength surface for the crushed material. Figure (3) shows these strength surfaces.

\section{b) Steel reinforcement modeling}

Under blast loading, the reinforcing steel may be subjected to strain hardening, strain rate hardening, and heat softening effects. Steel compression is approximately proportional to the pressure level. Thus, a linear EOS for steel is used [4]. The pressure level is dependent on the bulk modulus and the compression. In this study, a perfect (non-hardening) Von Mises model [2] is adopted to model the response of the steel bars in concrete.

\section{c) Soil modeling}

There are two kinds of soils involved in the problem under investigation; clay and sand. The clay is modeled with the elasto-plastic hydro material model. For clays the water content has a profound influence on ground shock propagation. When the saturation approaches one hundred percent, peak stresses and accelerations similar to shock wave propagation in free water have been observed [3]. Therefore, the shock EOS is used [4]. A linear relationship between shock velocity and particle velocity was defined. The Von Mises yield surface was defined as a strength model. A hydro tensile failure limit with a small negative pressure value was defined.

Since sand is a granular material, it is necessary to adopt a granular material model with Compaction EOS when sand is subjected to blast loads. Laine and Sandvik [7] had performed four tri-axial cylindrical tests and three tri-axial shear tests to derive the mechanical properties of sand utilized for this model. A hydro tensile failure is also used for the sand, and the hydro tensile limit is set to be $\mathrm{p} \min =-1 \mathrm{e}^{-3} \mathrm{~Pa}$ as the failure criterion [7]. This means that when the $\mathrm{p}_{\text {min }}$ is reached, the cell is not allowed to resist shear stresses.

\section{d) Explosion modeling}

The high explosive blast wave modeling facility in AUTODYN-2D is a capability that has only recently been exploited on the so-called remapping function. Remapping allows taking the solution of 2D analysis and impose it upon a selected region, of a 3D model where an extra physical dimension can be modeled. This procedure not only reduces the time required for a calculation but also increases its accuracy due to the fine $2 \mathrm{D}$ mesh resolution in the initial high explosive detonation and expansion phases. The explosive material at start time is modeled by the "Jones - Wilkins - Lee" (JWL) EOS, it can be written in the form [4]

$$
P=C_{1}\left(1-\frac{\omega}{r_{1} v}\right) e^{-r_{1} v}+C_{2}\left(1-\frac{\omega}{r_{2} v}\right) e^{-r_{2} v}+\frac{\omega e}{v}
$$

where $C_{1}, C_{2}, r_{1}$ and $r_{2}$ are constants and $e, \omega$ and $v$ are the internal energy, adiabatic constant and specific volume respectively, and its values for many common explosives have been determined from dynamic experiments and are available in AUTODYN material library [4]. 
From equation (1), it can be shown that at large expansion ratios the first and second terms on the right hand side of the equation become negligible and hence the behavior of the explosive tends towards that of an ideal gas. Therefore, at large expansion ratios, where the explosive has expanded by a factor of approximately 10 from its original volume, it is valid to switch the EOS for a high explosive from JWL to ideal gas EOS [4].

\subsection{Boundaries and interactions modeling}

To reduce the computational process, two symmetrical plans intersecting at the center of gravity of the charge were used to simulate buried structure subjected to blast wave. Nonreflecting boundaries (Transmit boundary conditions) are applied to simulate the semiinfinite domain of the soil extending away from the edges of the mesh to limit the reflection of stress waves, and free boundary surface is applied at the top (ground surface).

An accurate representation of the interface between the structure and the surrounding medium is a crucial to successful analysis of the structural response. According to Baylot [8], the soil-structure (concrete) interface strengths may be described by Coulomb failure laws. On a smooth soil-concrete interface failure is initiated when the shear parallel to the surface exceeds the failure law; whereas on a rough soil-concrete interface the failure is initiated when the maximum soil shear stress exceeds the failure law. The experimental results [8] indicate that the strength properties of the interface are close to the strength properties of soil. For this reason, in the present study the interface between the concrete and soil (smooth) is modeled using Lagrange-Lagrange impact slide surface facility in AUTODYN [4]. The explosive-soil interaction is simulated using 3D Euler-Lagrange coupling facility.

\section{Numerical application}

A proposed numerical model is used to simulate one of the field tests conducted by Baylot [9] to study the soil-structure interaction for a high explosive charge placed close to the wall of a buried structure. The structure was buried in carefully placed, nearly saturated clay soil. The assumed soil properties are: the initial density is $1900 \mathrm{~kg} / \mathrm{m}^{3}$, the shear modulus is $1.01 \mathrm{Gpa}$, and the yield stress is $400 \mathrm{Kpa}$. The explosive source consisted of $7 \mathrm{~kg}$ of C4 of cylindrical charge with 686- $\mathrm{mm}$ long and 90-mm diameter. The charge was placed with its midlength opposite to the middle of the test slab. The clear spans of the test slab are $1.0 \mathrm{~m}$ vertically and $4.0 \mathrm{~m}$ horizontally. The slab thickness is $220 \mathrm{~mm}$. A cross section through field test is shown in Figure (4). The top and bottom edges of RC test slab overlapped the RC reaction structure and were bolted on to provide a moment-resistant connection. The ends of the slab overlapped the reaction structure, but were not bolted. Material property test data are provided in Table (1). In these experiments, accelerometers and pressure sensors were placed in the soil at various ranges from the charge to provide free-field stress and motion measurements. Accelerometer records are integrated once to obtain free-field velocities and again to obtain displacements. Data were only reported for $20 \mathrm{msec}$.

To simulate the field test in the proposed model, eight nodes Lagrange elements are used to simulate concrete and soil materials, while eight nodes Euler elements are used for explosive material. The reaction structure is modeled by 19680 elements, the test slab is modeled by 5120 elements and the medium is modeled by 85508 elements. The steel reinforcing is modeled by two node beam elements. The charge area is modeled by 25600 elements, the entire subgrid is initially filled with air at ambient conditions, and as mentioned 
in article (2.1-d), the explosion expressed in 2D will remapped to 3D charge area. The mesh details and material location of a system are shown in Figure (5).

The displacement time history for the center of both the slab and the floor are shown in Figure (6). The deformation of test slab can be obtained by subtracting the second from the first and it will be as shown in Figure $(7)$. Figures $(8,9)$ show the comparison between the results obtained from the proposed model with that obtained from test results and Baylot [9], it is clear that the proposed model agree well with the field test results. A summary of the peak values are presented in Table (2). Figure (10) shows material status and crater formation resulting from the explosion at different times. The pressure contours on the buried structure are shown in Figure (11). The damage location of test slab is shown in Figure (12).

\section{Effect of surrounding medium on buried structure response}

The proposed model is used to study the effect of medium on buried structure response. The demonstrated system in the numerical application is applied in sandy medium. The initial properties chosen for sand are based on the typical practical and laboratory tests for sand site, including, dry mass density $1671 \mathrm{~kg} / \mathrm{m}^{3}$, shear modulus $150 \mathrm{Mpa}$, friction angle $35^{\circ}$ and damping $3 \%$ [7].

Figures $(13,14)$ show the comparison between interface pressure time history and impulse time history at the center of the test slab in two mediums. The displacement time history at the center of test slab in nearly saturated clay and sand mediums are shown in Figure (15), the maximum displacement reaches $39 \mathrm{~mm}$ in clay while the corresponding value is $7 \mathrm{~mm}$ in sand. Figure (16) shows the damage percentage (function of effective plastic strain) with time in two mediums. Summary of peak responses is presented in Table (3), which shows the efficiency of sand medium to absorb blast energy.

\section{Ground shock attenuation}

When a layer with lower impedance is introduced in front of the wall facing the explosion, a relief wave will generated. This relief wave disturbs the incident pressure. This is a very positive effect that decreases the total load of the ground shock. Sand has been recognized as a good attenuation layer and a good material to absorb blast energy. However, in this section the following questions will be answered: how much will the maximum pressure decrease when sand in the front of the underground wall is utilized?, What thickness should the sand layer have for optimum structural response reduction?

Dry sand layers with thicknesses: 10, 20, 30, 40, 50, and $60 \mathrm{~cm}$ were utilized as backpacking material behind the slab facing the explosion demonstrated in numerical application. In all cases the depth of charge is assumed to be at the same horizontal level as the center of test slab at $(1.5 \mathrm{~m})$ from the ground surface, and a stand off distance is $(1.5 \mathrm{~m})$.

The reduction in the peak interface pressure at the front slab center is shown in Figure(17), Figures $(18,19)$ show the reduction of peak particle velocity (PPV) and peak displacement at the center of test slab respectively. It is clear that, the sand layer should be at least $30 \mathrm{~cm}$ to achieve $45 \%$ reduction in PPV and $55 \%$ in peak displacement for the studied load. 


\section{Conclusions}

A proposed full coupled numerical approach for simulating ground shock and the response of buried structure subjected to subsurface blast loading is presented in this paper. The model overcomes many difficulties that are known to be associated with other coupling methods. The proposed approach makes use of various state-of-the-art material models to enhance the reliability of the simulation results. The numerical example shows that the proposed model is capable to reproduce the physical processes in a realistic manner. The computed peak stresses and buried structure response are in excellent agreement with available experimental data. With this full coupled model, a wide range of problems related to the effects of subsurface explosions on buried structures can be investigated numerically.

Added protection should be considered when the structure is buried in dense or saturated soils. The improvement of a buried structure to withstand ground shock from subsurface explosion can be achieved by introducing a sand layer as attenuation backpacking material behind the slab facing the explosion; the thickness of the layer should be at least $30 \mathrm{~cm}$ to achieve approximately $45 \%$ and 55\% reduction in PPV and peak displacement respectively for the studied case. To achieve the general optimum thickness of sand layer for other load cases needs further studies.

\section{References}

[1] Weidlinger P., and Hinman, “Analysis of Underground Protective Structures”, Journal of Structural Engineering, Vol.114, No. 7, July, pp 1658-1673, 1988.

[2] Krauthammer T. and O'Daniel J. L., "Assessment of Numerical Simulations Capabilities for Medium-Structure Interaction Systems under Explosive Loads", Computers \& structures, Vol. 63, No. 5, pp. 575-887, 1997.

[3] Technical Manual TM5-885-1, "Fundamentals of Protective Design for Conventional Weapons", Headquarters Department of the Army, Washington, DC, 1986.

[4] AUTODYN Theory Manuals, Version 4.3, Century Dynamics, Inc.2003 Sam Ramon, USA, 2003.

[5] Benson D. J., “Computational Methods in Lagrangian and Eulerian Hydrocodes”, Comp Meth Appl Eng, Vol. 99, pp. 235-394, 1992.

[6] Riedel W., Thoma K., Hiermaier S., "Numerical Analysis Using a New Macroscopic Concrete Model for Hydrocodes ”, Proceedings of 9th International Symposium of the Effects of Munitions With Structures, 1999.

[7] Laine L. and Sandvik A., "Derivation of Mechanical Properties for Sand", $4^{\text {th }}$ AsiaPacific Conference on Shock \& Impact Loads on Structures, pp 361-368, Nov. ,2001.

[8] Baylot J. T., "Parameters Affecting Loads on Buried Structures Subjected to Localized Blast Effects", Final Report A91-LD-003,Army Waterways Experiment Station, Corps of Engineering, Vicksburg, 1992.

[9] Baylot J. T., "Effect of Soil Flow Changes on Structure Loads", Journal of Structural Engineering, Vol.126, No. 12, December, pp 1434-1441, 2000. 
Table 1- Test slab parameters [9]

\begin{tabular}{|l|l|c|}
\hline Material & \multicolumn{1}{|c|}{ Parameter } & Value \\
\hline \hline \multirow{4}{*}{ Concrete } & Thickness (mm) & 220 \\
\cline { 2 - 3 } & Effective depth & 196 \\
\cline { 2 - 3 } & Compressive strength (MPa) & 44 \\
\hline \multirow{2}{*}{$\begin{array}{l}\text { Vertical steel } \\
\text { (each face) }\end{array}$} & Spacing (mm) & 76.2 \\
\cline { 2 - 3 } & Bar diameter (mm) & 9.5 \\
\cline { 2 - 3 } & Yield strength (MPa) & 465 \\
\hline \multirow{2}{*}{$\begin{array}{l}\text { Horizontal steel } \\
\text { each face) }\end{array}$} & Spacing (mm) & 400.4 \\
\cline { 2 - 3 } & Bar diameter (mm) & 4 \\
\cline { 2 - 3 } & Yield strength (MPa) & 401 \\
\hline
\end{tabular}

Table 2- Comparison of the peak values at the center of the test slab

\begin{tabular}{|l|c|c|c|}
\hline Parameter & Filed Test & Baylot & Autodyn-3D \\
\hline \hline Peak interface stress [MPa]. & 17.1 & 18.2 & 16.2 \\
\hline Peak deformation at slab center [mm]. & 40 & 36 & 38.22 \\
\hline Time of peak response [msec]. & 10.7 & 9.8 & 11.2 \\
\hline
\end{tabular}

Table 3- Comparison between peak responses in two different mediums

\begin{tabular}{|l|c|c|}
\hline Parameter & Clay medium & Sand medium \\
\hline \hline Peak interface stress at the center of test slab [MPa]. & 16.2 & 1.91 \\
\hline Peak impulse at front slab/unite area, [MPa-msec] & 16.85 & 6.24 \\
\hline PPV of the center of test slab, [m/s] & 8.74 & 2.15 \\
\hline Peak displacement of the center of test slab, [mm] & 39 & 7 \\
\hline Peak displacement at upper support, [mm] & 1.57 & 2.1 \\
\hline Peak displacement at lower support, [mm] & 1.94 & 2.2 \\
\hline
\end{tabular}




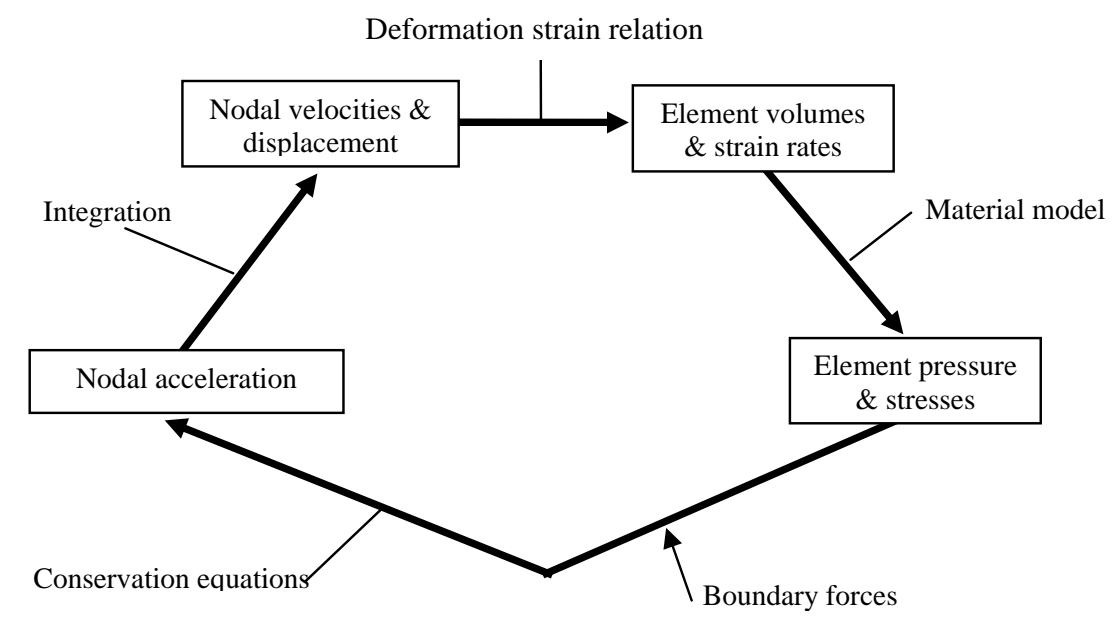

Fig. (1)- Illustration of computational cycle in a proposed model.

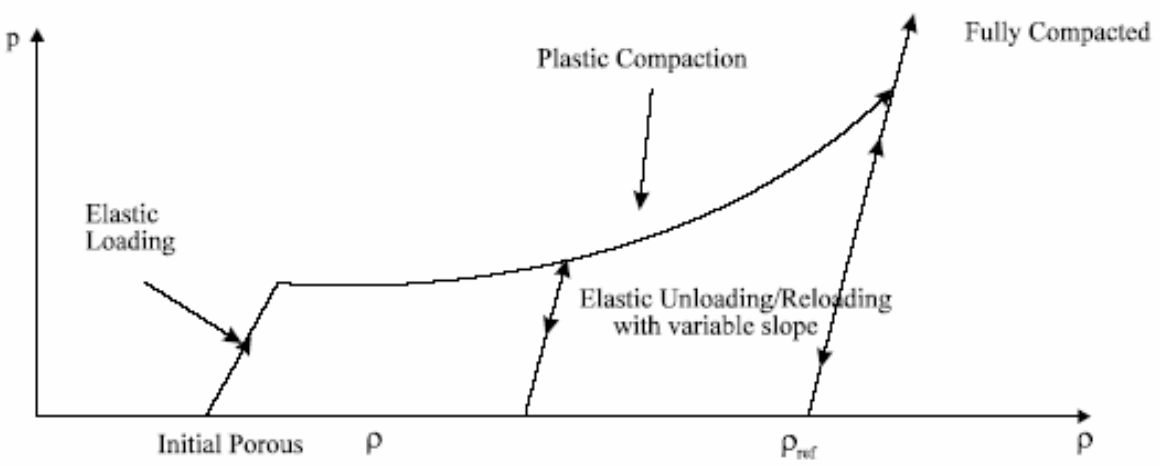

Fig. (2)- Combined P-Alpha and polynomial (EOS) for brittle materials.

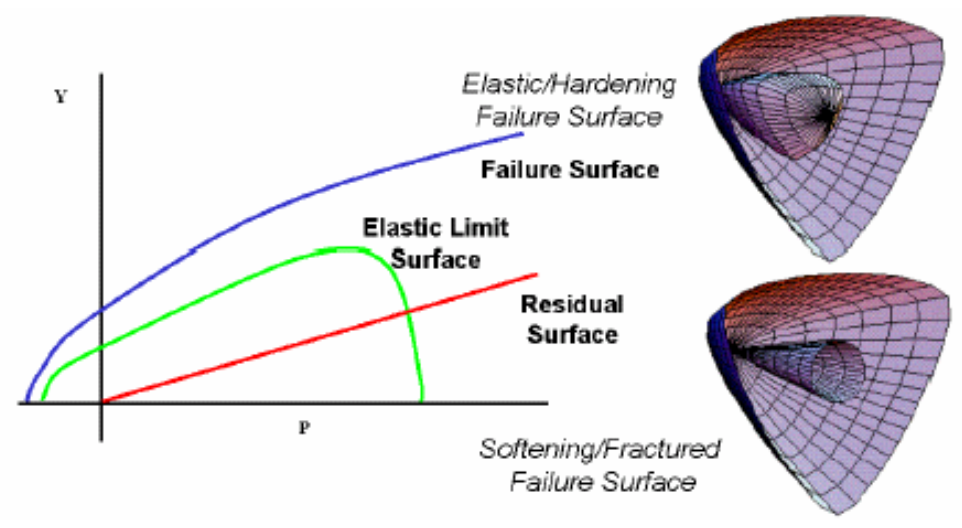

Fig. (3)- RHT model for brittle materials. 
Ground surface

- Target points

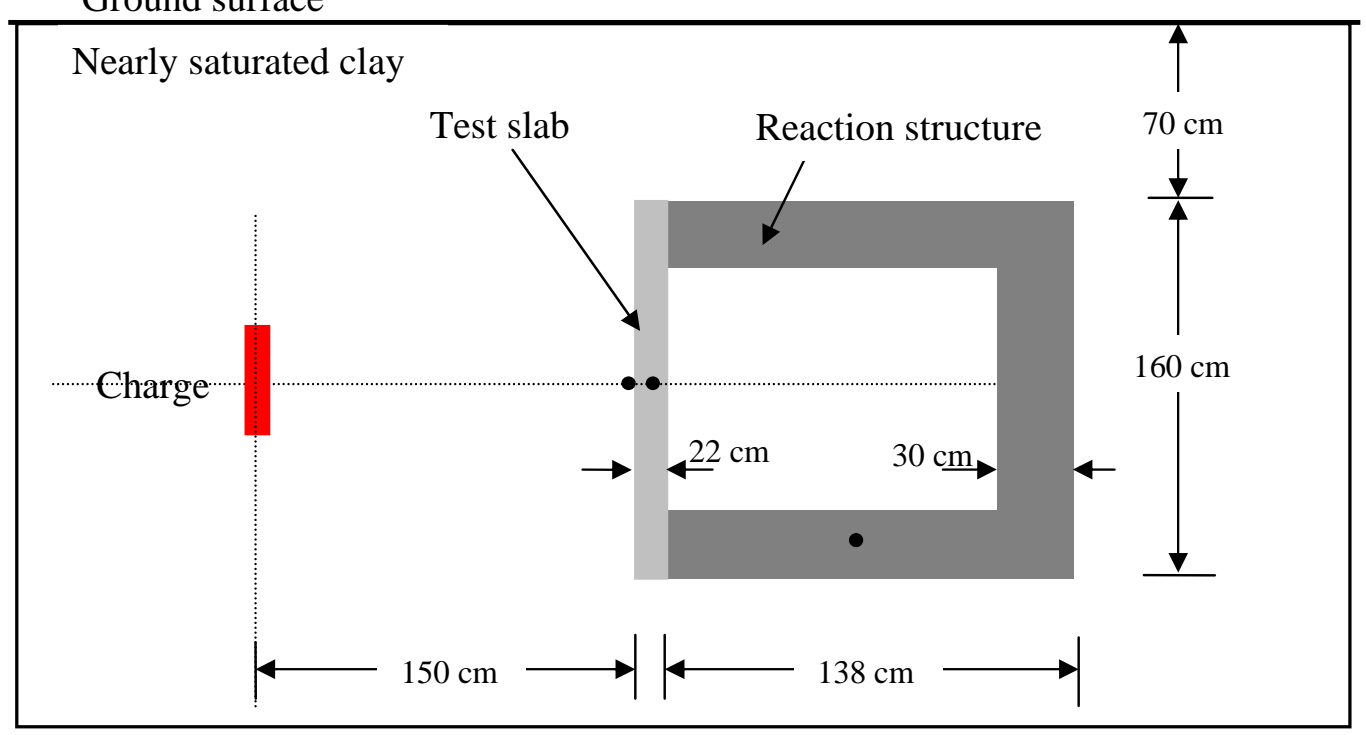

Fig. (4)- Cross section through filed test [9].

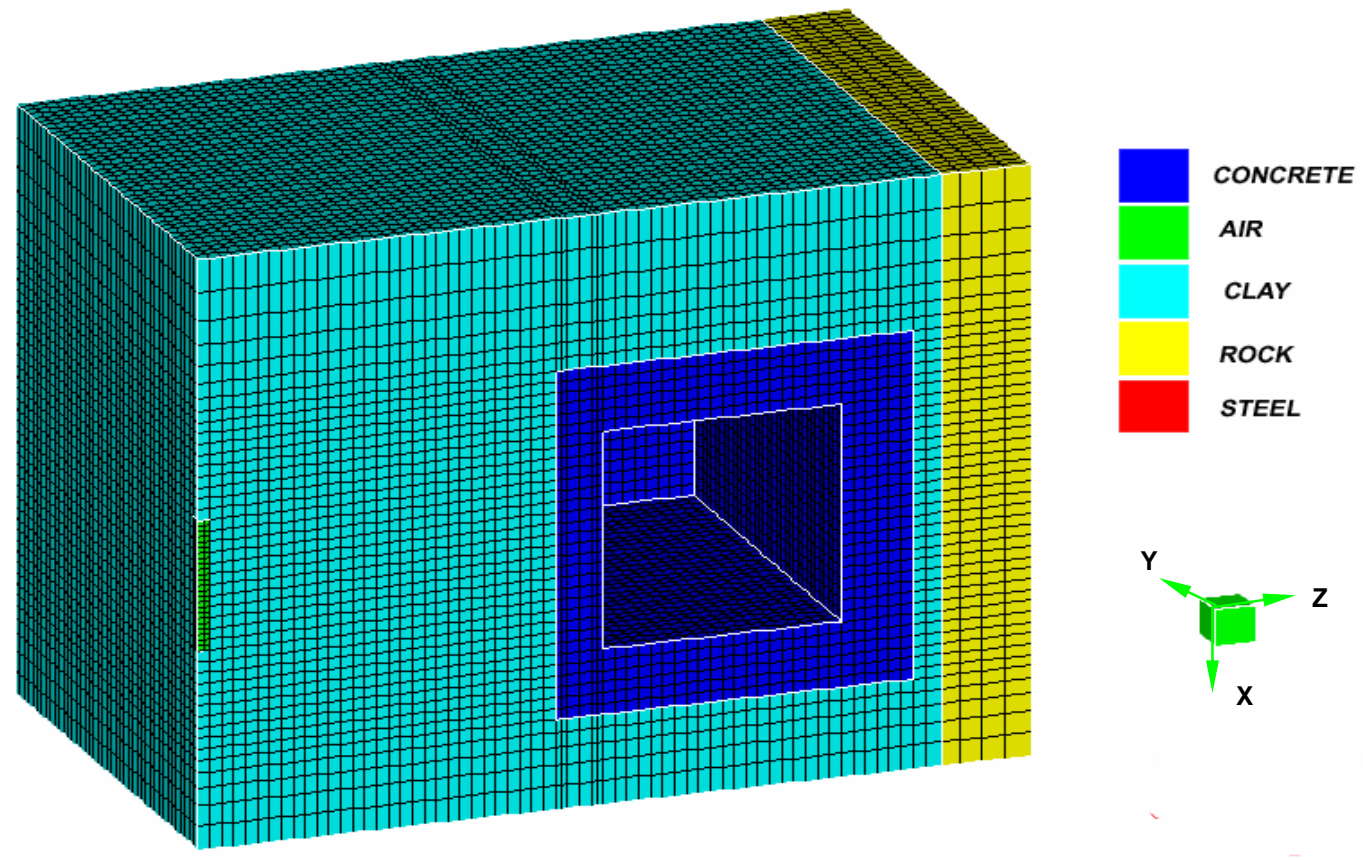

Fig. (5)- Material location and mesh details. 


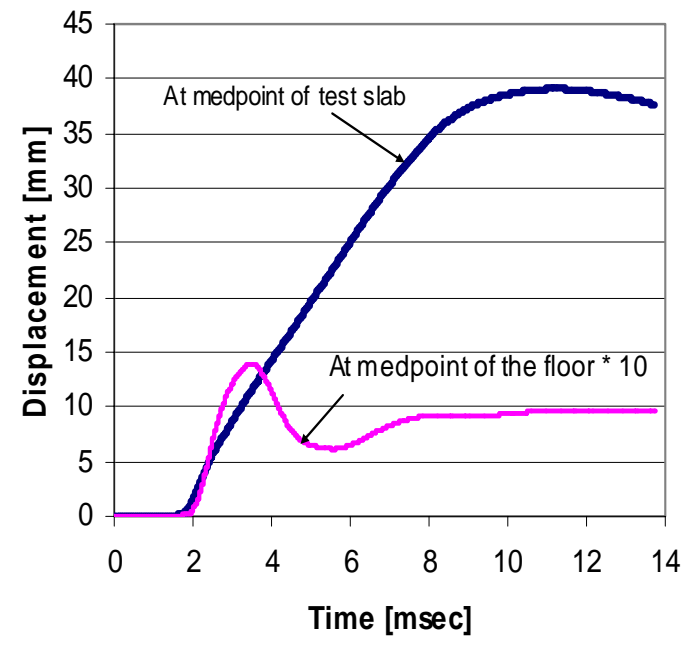

Fig. (6)- Displacement time histories of test slab and floor.

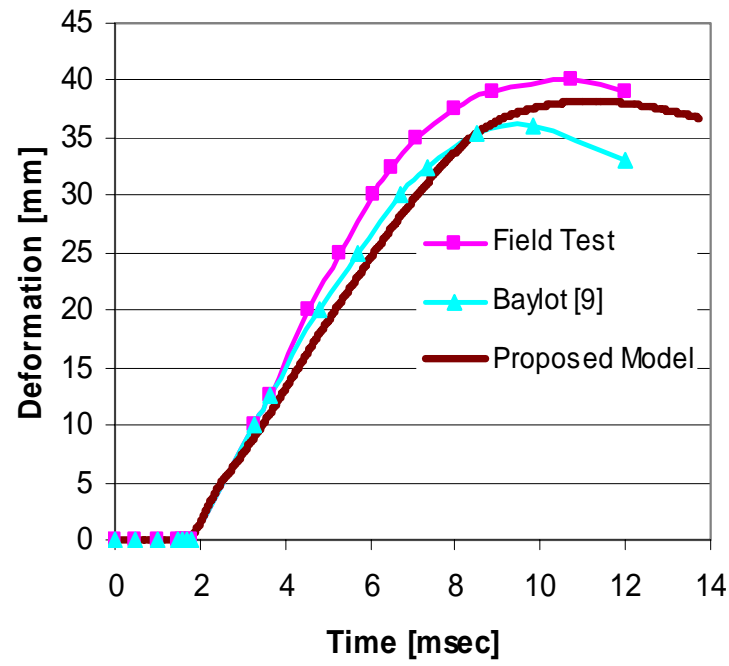

Fig. (8)- Comparison between deformation time histories at the center of test slab.

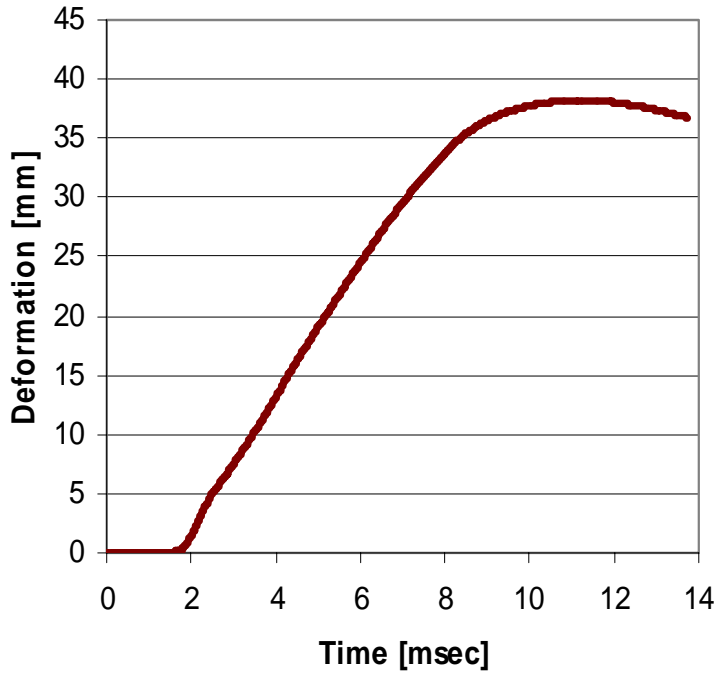

Fig. (7)- Deformation time history at the center of test slab.

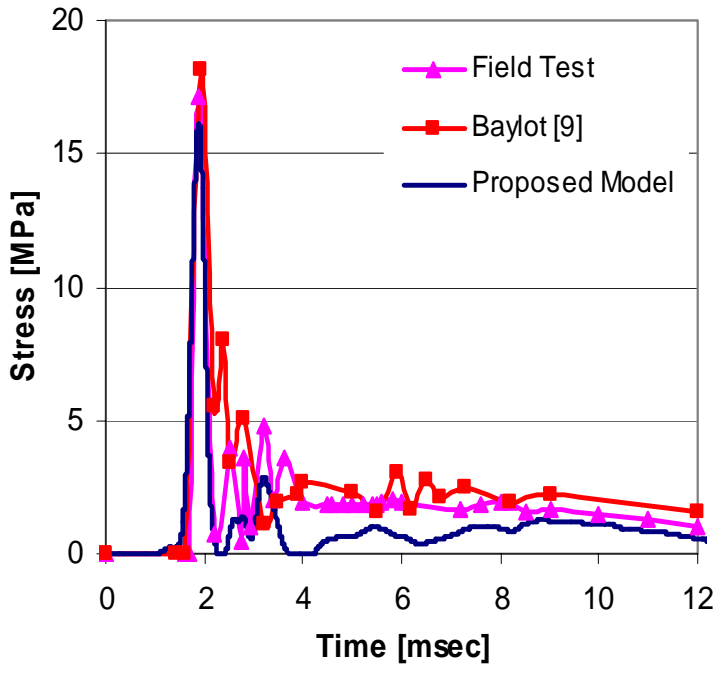

Fig. (9)- Comparison between interface stress time histories at the center of test slab. 


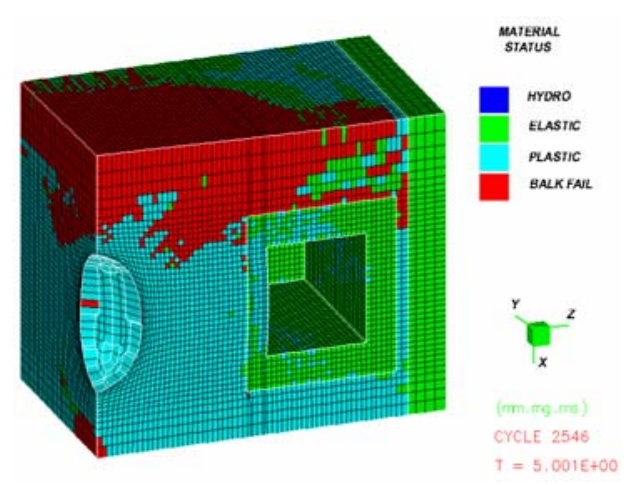

At 5 msec

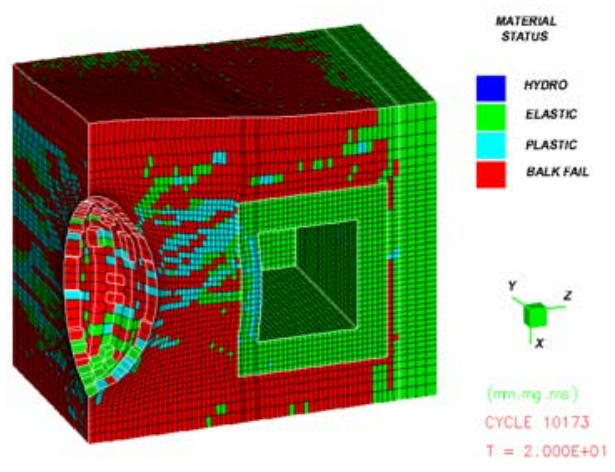

At 20 msec

Fig. (10)- Material status and crater formation.

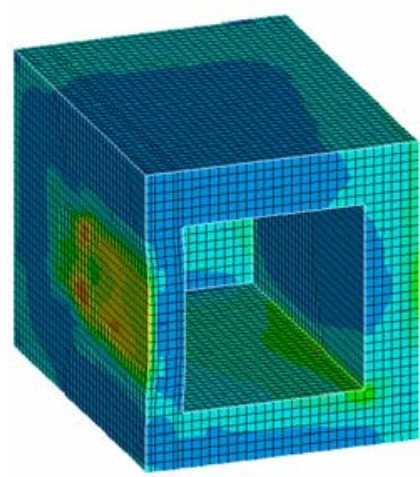

At 5 msec

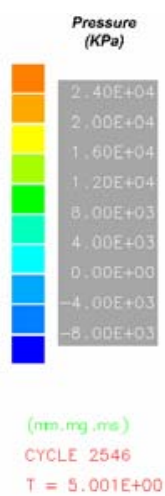

$T=5.001 E+00$

Fig. (11)- Pressure contours on buried structure at different times.
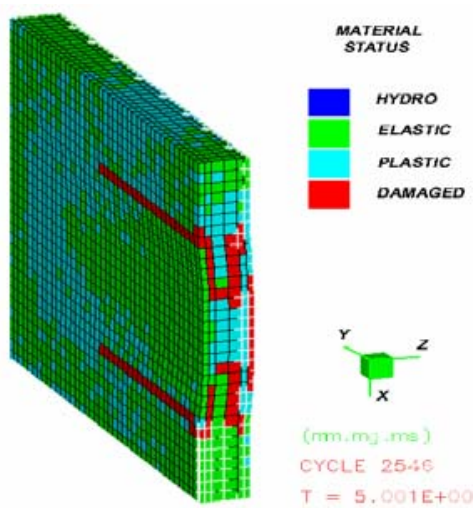

At 5 msec
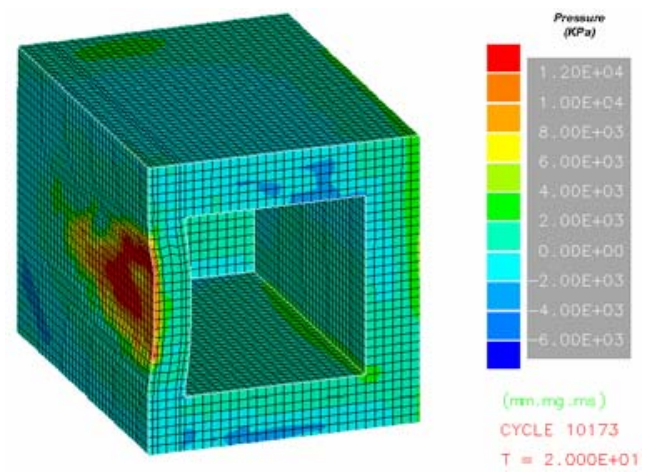

At 20 msec 


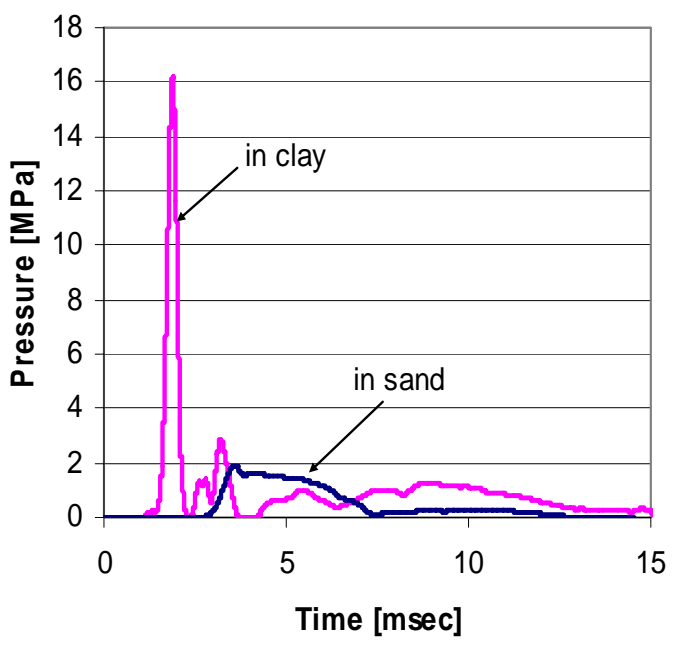

Fig. (13)- Comparison between interface stress time histories at the center of test slab.

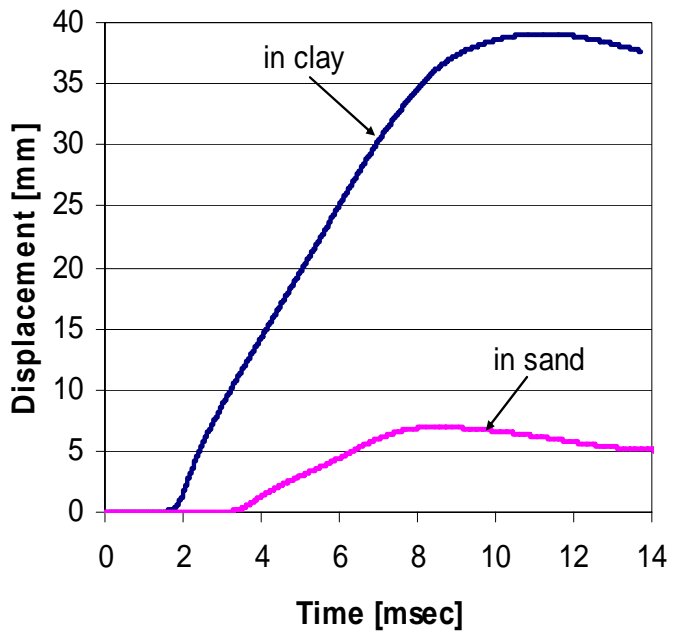

Fig. (15)- Comparison between displacement time histories of the center of test slab.

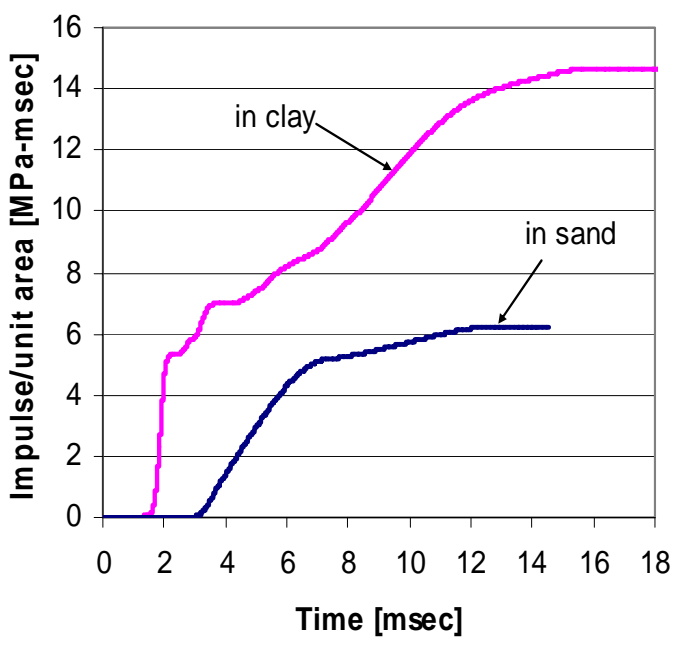

Fig. (14)- Comparison between impulse time histories at the center of test slab.

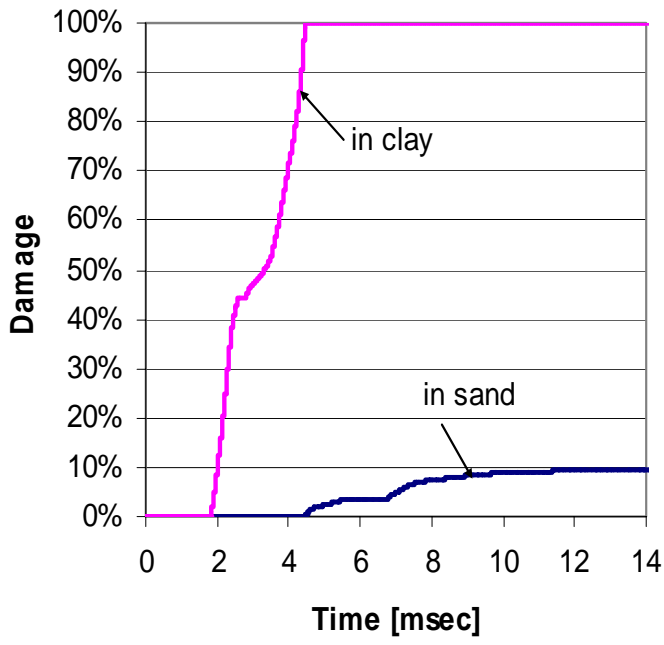

Fig. (16)- Comparison between damage time histories at the center of test slab. 

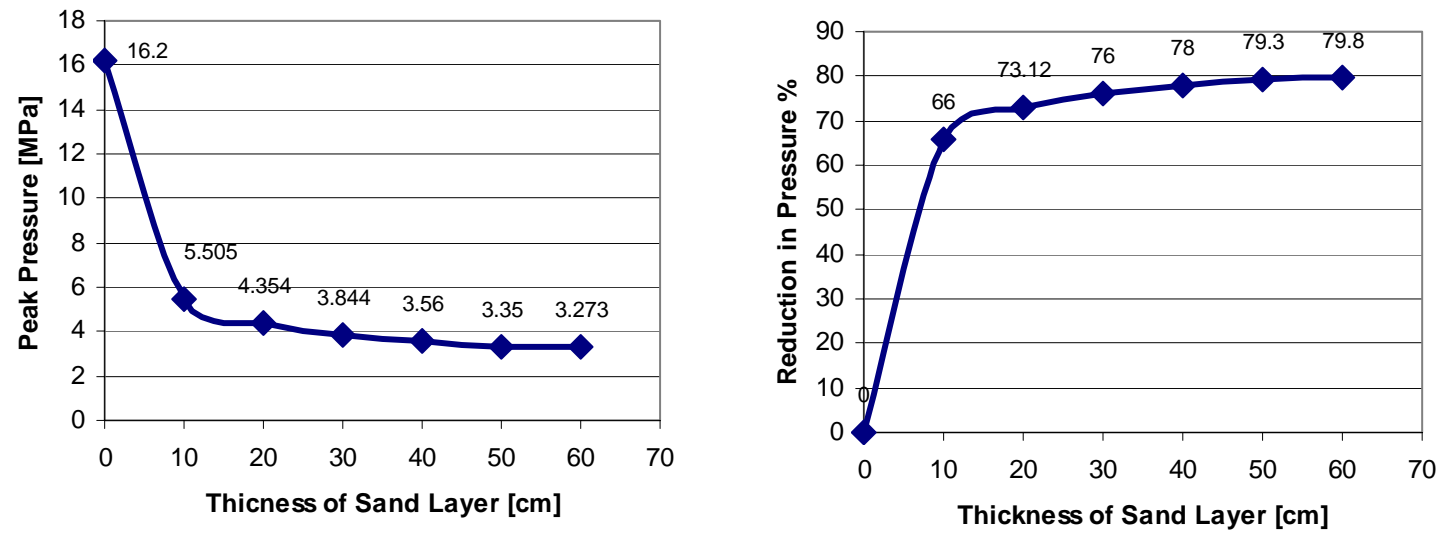

Fig. (17)- Reduction of peak interface pressure at the center of test slab with increasing of sand layer.
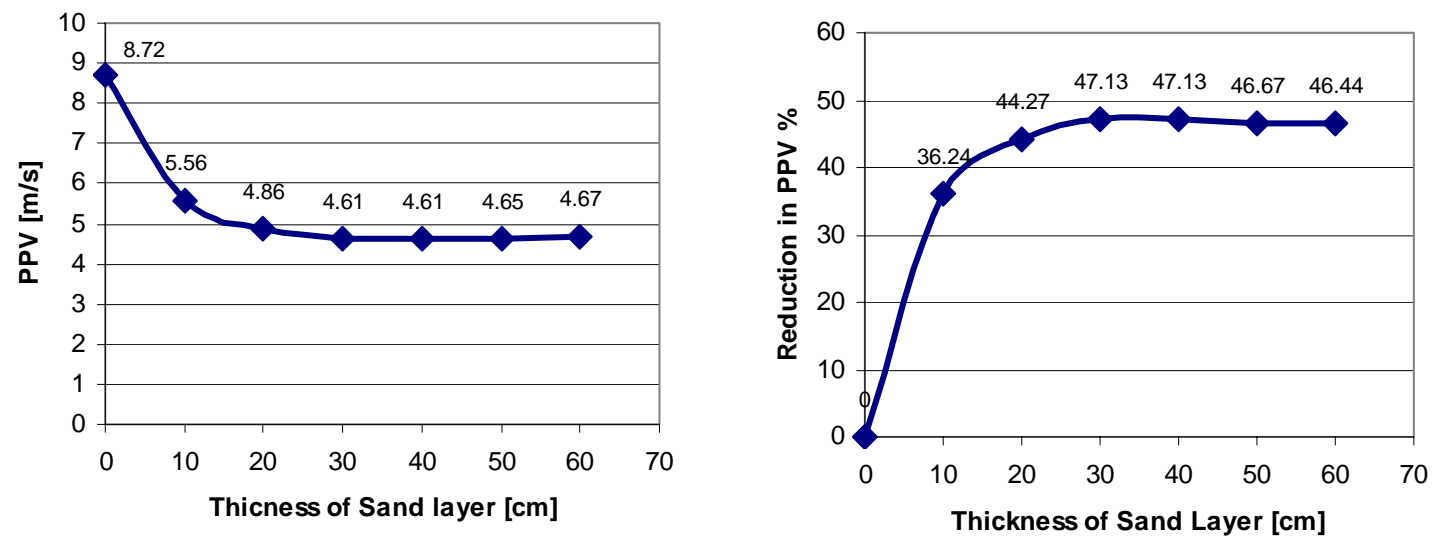

Fig. (18)- Reduction of PPV of the center of test slab with increasing of sand layer.
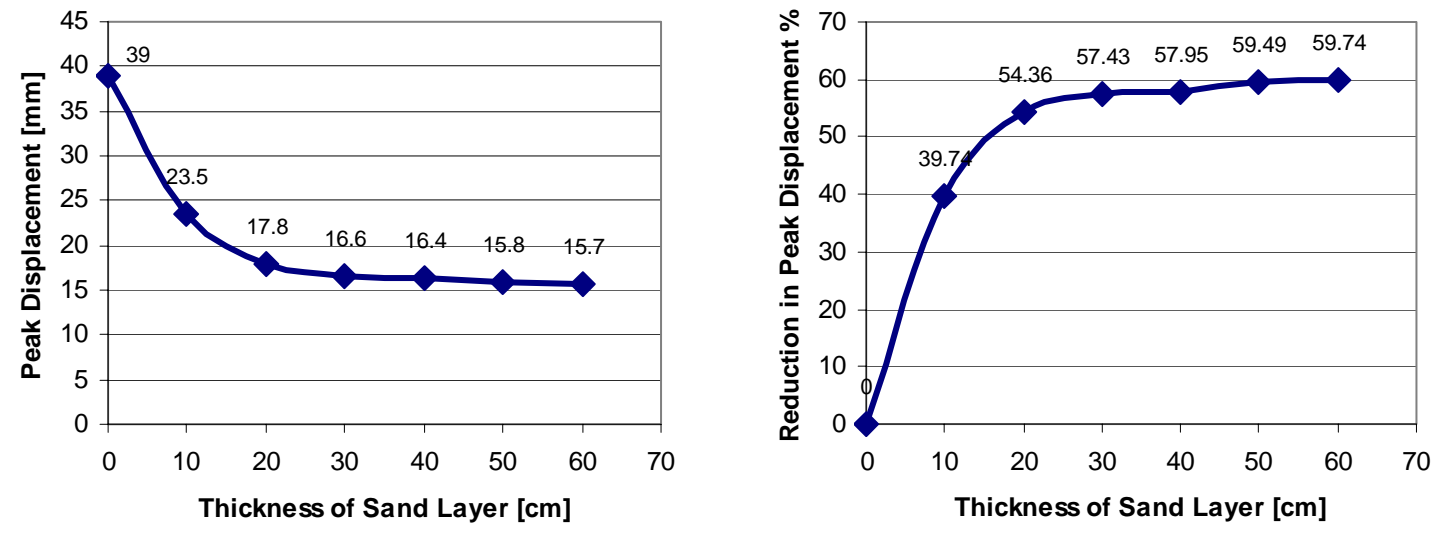

Fig. (19)- Reduction of peak displacement of the center of test slab with increasing of sand layer. 